\title{
Intraoperative hypotension is associated with acute kidney injury in non-cardiac surgery
}

Linn Hallqvist, Fredrik Granath and Max Bell

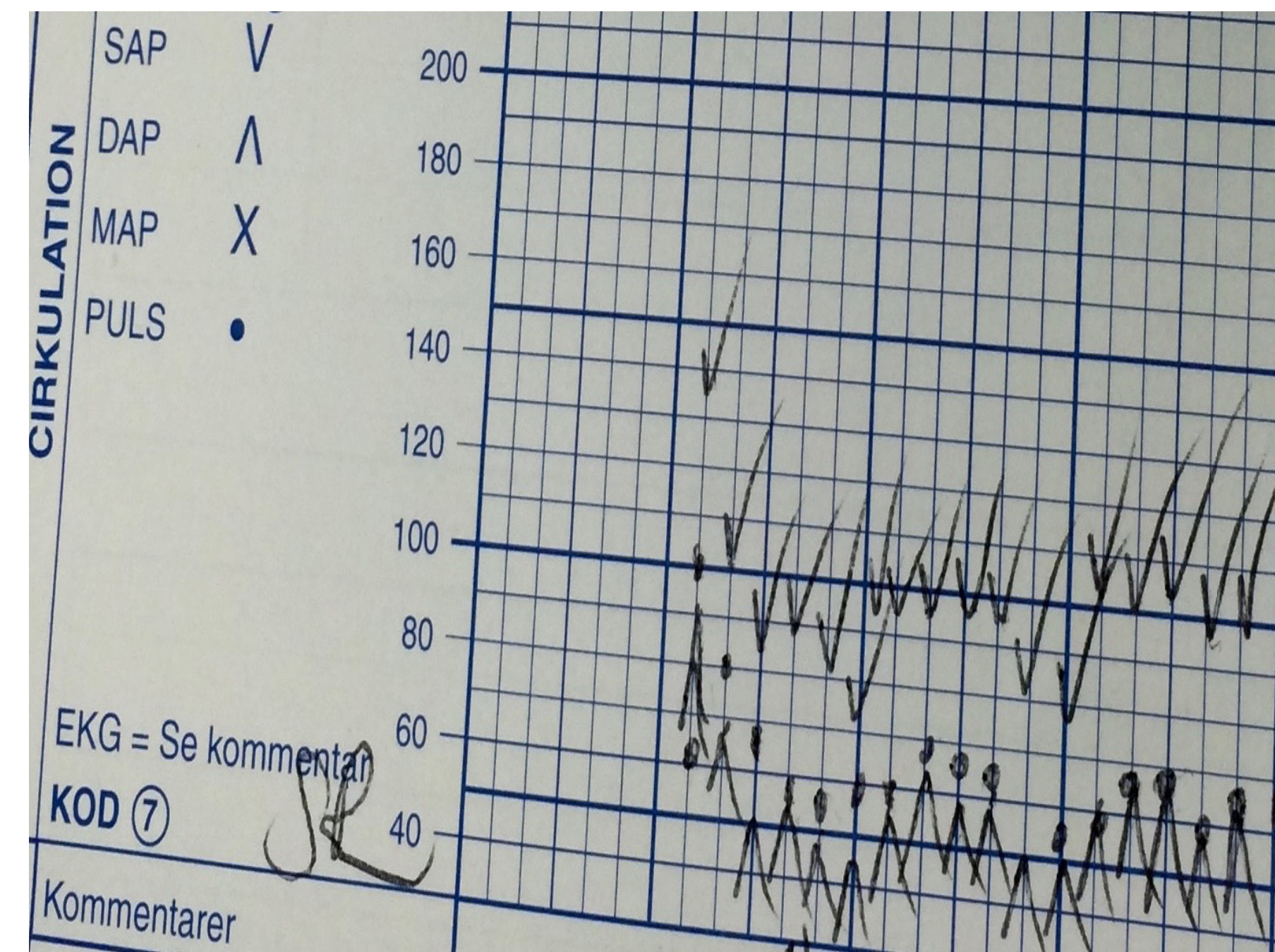

\section{Background}

Postoperative acute kidney injury (AKI) is associated with increased mortality and other postoperative complications.

\section{Objectives}

The present study sought to investigate if individually based intraoperative hypotension, and other potential risk factors, including; comorbidites, blood loss, fluid balance etc, were associated with increased risk of perioperative AKI.

\section{Methods}

In this observational cohort study of patients undergoing major elective non-cardiac surgery, who required an overnight admission at the postoperative unit, at the Karolinska University Hospital, Stockholm, Sweden during 2012-13 and 2015-16, preoperative risk factors (comorbidities), intraoperative events (hypotension defined as a decrease in systolic blood pressure relative each patient's baseline lasting $>5 \mathrm{~min}$ ) and postoperative data were recorded. Levels of creatinine were measured before surgery and on postoperative day 1 to 3 . AKI was defined according to the KDIGO criteria.

\section{Conclusions}

In major non-cardiac surgery, there is an association between intraoperative fall in systolic blood pressure $>50 \%$ from baseline and postoperative acute kidney injury (AKI).

\section{Results}

Of the final cohort of 470 AKI incidence patients, 127 (27\%) developed AKI within 2 postoperative days. Multivariable logistic regression analyses demonstrated that an intraoperative hypotensive event $>50 \%$ was associated with a more than doubled risk of postoperative AKI (OR, 2,46; 95\% CI 1.31-4,62).
Preoperative predictors and odds ratios of $\mathrm{AKI}$ in relation to an intraoperative hypotensive event* and the influence of intraoperative blood loss.

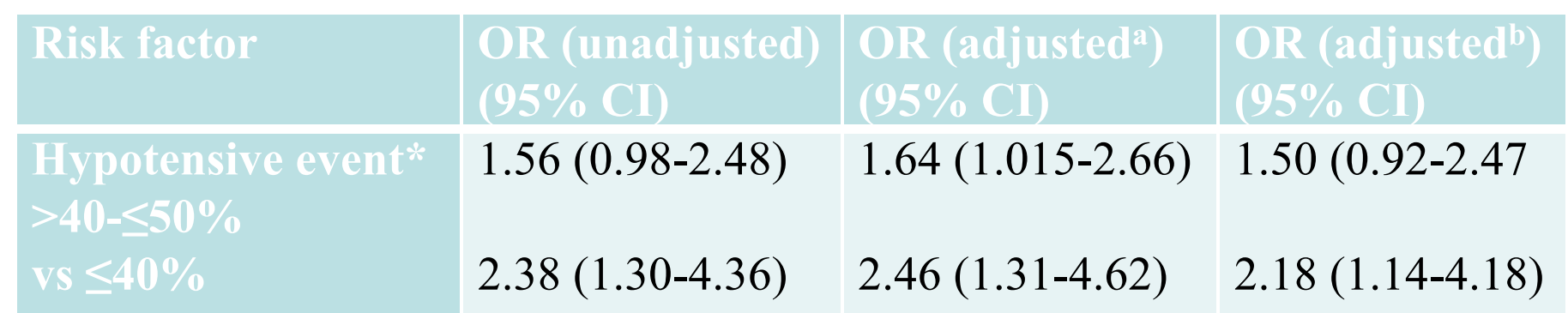

* Decrease in systolic blood pressure in percent relative to baseline for $>5$ min. ${ }^{a}$ Adjusted for the covariates: gender (male), ASA $>2$, treated hypertension and pre-operative creatinine $>90 \mu \mathrm{mol} / \mathrm{L}$.

b Adjusted for the covariates mentioned above and blood loss in quartiles. 